\title{
Research Paper \\ Comparing Gait Variability Between Deaf and Normal-Hearing Children After Proprioception Training
}

\author{
Fatemeh Yaghoubi Hamraz ${ }^{1}$ (D), ${ }^{*}$ Mahdi Majlesi $^{1}$ (])
}

1. Department of Sport Biomechanics, Faculty of Humanities, Hamadan Branch, Islamic Azad University, Hamadan, Iran.

Citation: Yaghoubi Hamraz F, Majlesi M. [Comparing Gait Variability Between Deaf and Normal-Hearing Children After Proprioception Training (Persian)]. Journal of Sport Biomechanics. 2020; 5(4):262-271. https://doi.org/10.32598/biomechanics.5.4.6

dol'https://doi.org/10.32598/biomechanics.5.4.6

Key words:

Gait, Deafness,

Spatio-temporal variables.

\section{ABSTRACT}

Article Info:

Received: 10 Sep 2019

Accepted: 23 Jan 2020

Available Online: 01 Mar 2020

\section{Extended Abstract}

\section{Introduction}

H

earing loss is one of the most common sensory disorders in developed countries [1, 2] and is usually diagnosed in the first years of life. Three sensory systems are involved in controlling balance and posture, including visual, proprioceptive and vestibular systems. Among these, the vestibular system, in addition to control, plays an important role in human interaction with the environment [6]. Although various studies have focused on the analysis of gait in individuals with hearing loss [9, 17], biomechanical variables are not commonly considered in their activities including gait. Walking is a complex task that requires functional coordination of various biomechanical variables. Modification of walking techniques has recently been recommended as a part of rehabilitation programs [20].

Therefore, the biomechanical variables related to walking are of clinical importance and are used to provide feedback that can be used to evaluate the therapeutic effects or to plan

\section{* Corresponding Author:}

Mahdi Majlesi, PhD.

Address: Department of Sport Biomechanics, Faculty of Humanities, Hamadan Branch, Islamic Azad University, Hamadan, Iran.

Tel: +98 (918) 4077540

E-mail: majlesi11@gmail.com 
rehabilitation programs. Despite assessments of balance, movement disorders, and factors related to health and quality of life, the gait of these individuals has not yet been properly examined [15]. Therefore, it is necessary to evaluate the kinematic pattern and gait variability of deaf children. The aim of this study was to analyze the gait variability of deaf people in comparison with healthy people at mediolateral and anterior-posterior directions during walking.

\section{Methods}

The study population consists of all deaf and normal-hearing people living in Hamedan. Among them, 10 deaf people (aged 8-14 years) and 10 normal-hearing peers with similar age and anthropometric characteristics volunteered to participate in the study. The 3D Vicon motion analysis system was used to measure the kinematic gait variables in both groups. After the pre-test assessment, subjects participated in the proprioception training program for 4 weeks, 3 sessions per week each for 45 minutes with an emphasis on the proprioceptive system. After the training period, a post-test assessment was performed. In this study, the spatio-temporal gait parameters were extracted using Polygan version 3.5.2 software, and the formula (coefficient of variation) was used to calculate the variability of these parameters. Paired t-test was used for within-group comparison and independent t-test was used for between-group comparison. All statistical analyses were performed in SPSS software by considering a significance level of $(\mathrm{P}<0.05)$.

\section{Results}

The results of within-group comparison showed that variability in parameters cadence and gait speed, stride length and step length, stride time, step time, double-leg support time, single-leg support time, opposite leg support time and swing time was not significantly different before and after proprioception training. This indicates that gait variability was not affected between groups after exercise and the difference between the two groups was not significant $(\mathrm{P}>0.05)$.

\section{Discussion}

Most children who have vestibular disorder are not diagnosed because they have the ability to walk [23], but these children do not participate in games and outdoor activities, and teachers often complain of poor balance and coordination in these children that may prevent them from performing ideally [24]. In the present study, it was shown that the difference between the deaf and control groups when walking in normal position was high, but not significant. How- ever, when walking, the gait speed decreased significantly in deaf children compared to healthy peers.

Proprioception training could not have a significant effect on gait speed and cadence, but the difference between the two groups after exercise was reduced; however, deaf children still had a slower gait speed after exercise therapy. Despite assessments of balance, movement disorders, and factors related to health and quality of life, there is still no educational program for these children except in cases where neurological and orthopedic injuries have been identified [15]. The results of several studies consistent with these results have shown that the gait speed in deaf people is slower than in healthy people $[9,25,26]$. Studies have also shown that deaf people have poor cognitive function, especially executive function $[27,28]$. The association between impairment in executive function and gait has been confirmed by several studies [29-31] which is clearer during dual-task walking.

\section{Ethical Considerations}

\section{Compliance with ethical guidelines}

All subjects voluntarily participated in the present study after signing a consent form.

Funding

This research did not receive any specific grant from funding agencies in the public, commercial, or not-profit sectors.

Authors' contributions

All authors contributed in preparing this article.

Conflicts of interest

The authors declared no conflict of interest 
This Page Intentionally Left Blank 


\section{ارزيابى تغيير يذيرى كامبردارى افراد ناشنوا و مقايسه آن با افراد شُنوا يس از يك دوره تمرينات حسى -عمقى}

فاطمه يعقوبى همراز' (1). "مهدى مجلسى

ا. كروه تربيتبدنى و علوم ورزشى، دانشكده علوم انسانى، واحد هملان، دانشكاه آزاد اسلامى، همدان، ايران.

\section{حكيد}

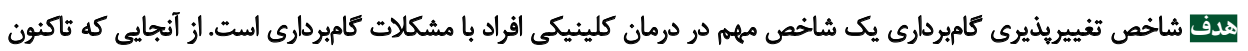

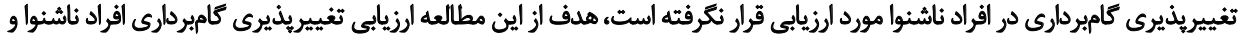
مقايسه آن با افراد سالم اسيت.

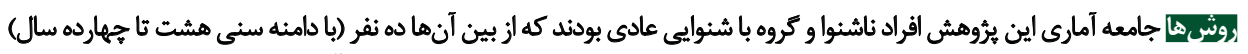

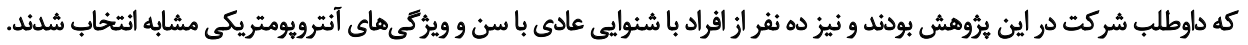

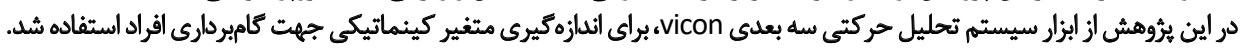

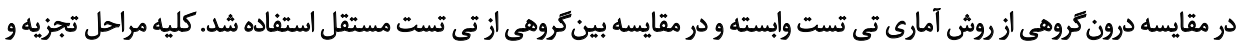

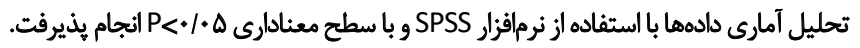

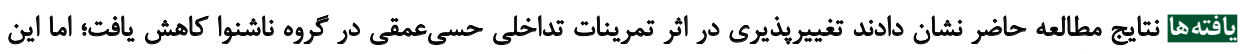

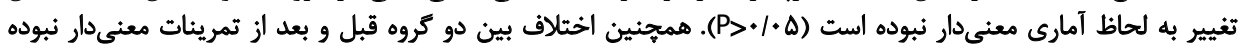

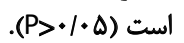

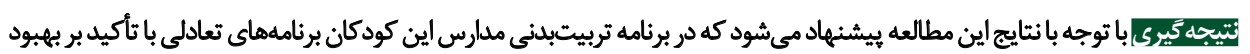

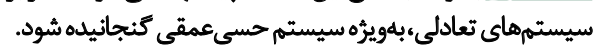

اطلاعات مقاله:

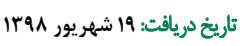

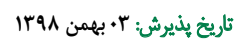

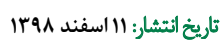

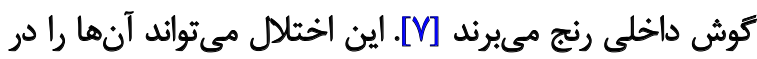

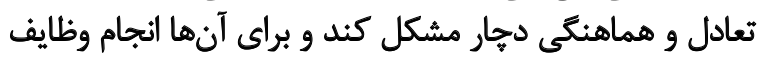

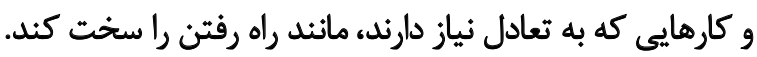

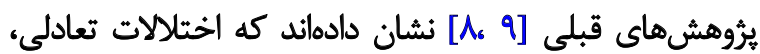

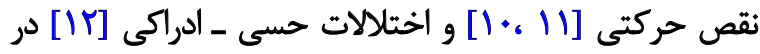

كودكان مبتلا به ناشنوايى شديد و مادرزادى وجود دارد.

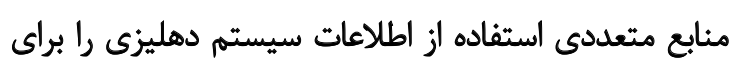

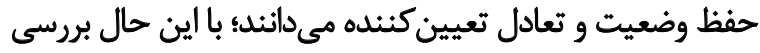

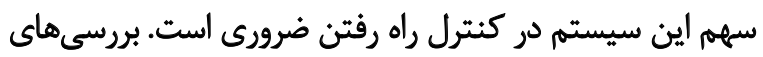

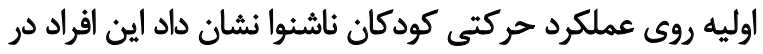

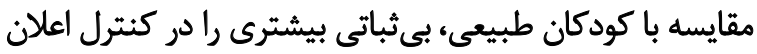

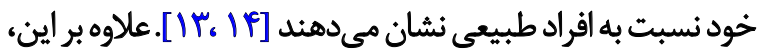

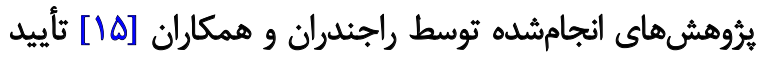

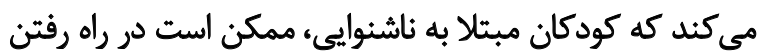

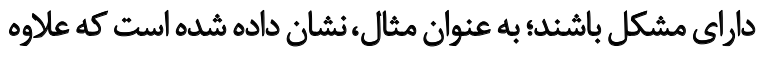

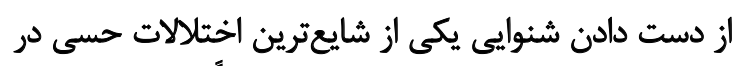

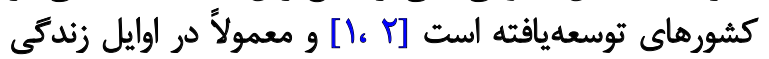

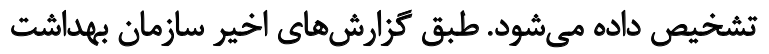

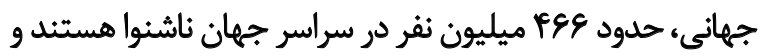

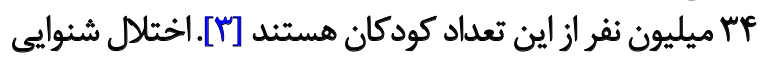

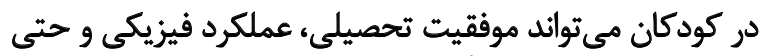

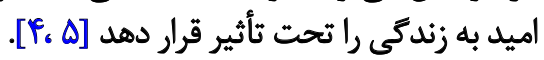

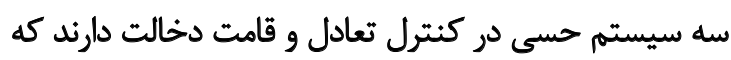

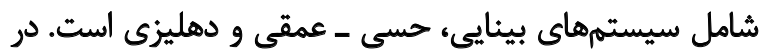

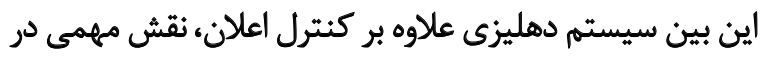

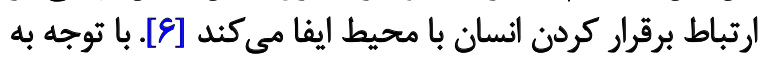

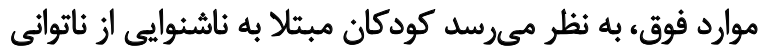

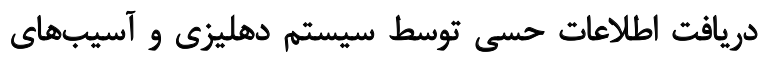

* نويسئده مسئول:

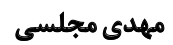

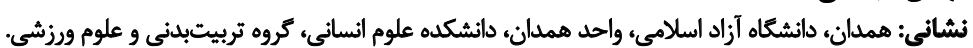

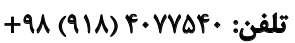
majlesi11@gmail.com يست الكترونيكي 
داشت، كمتر از / / ميلىمتر بود. براي اين منظور ابتدا دوربينها

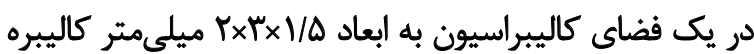

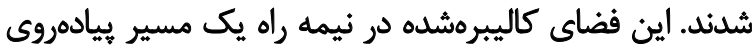

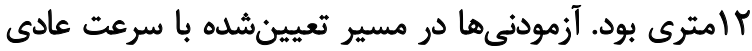

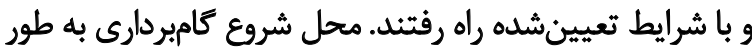

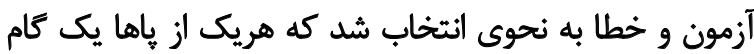

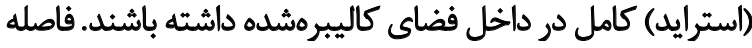

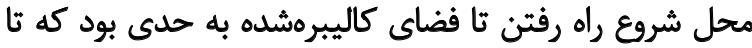

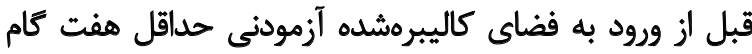

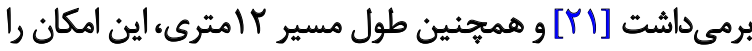

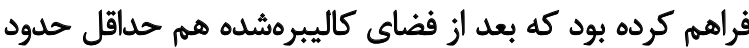

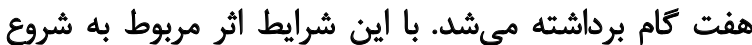
كاميردارى و توقف، حذف شد شد.

در اين بُروهش تست راه رفتن، قبل و بِ إز تمرينات تداخلى إنى

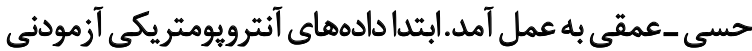

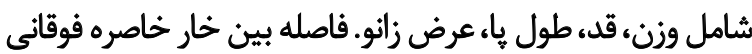

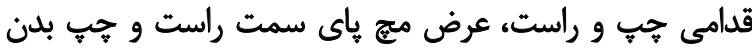

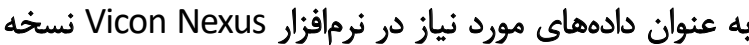

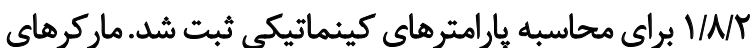

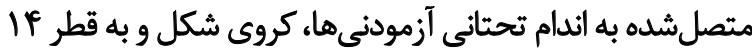

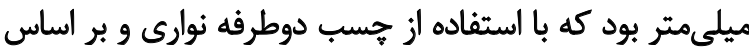

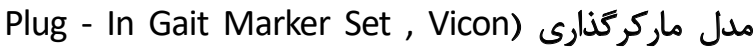

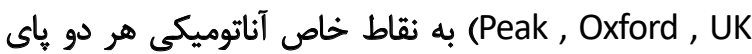
شركت كنئدكان متصل شد.

يس از كاليبراسيون دوربينها و نصب ماركرها ابتدا آزمودنىها

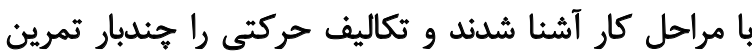

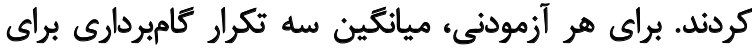

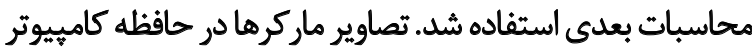

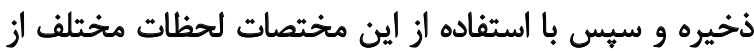

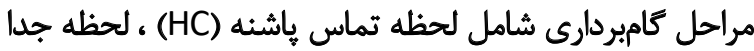

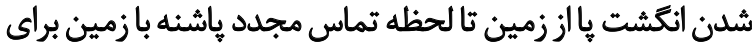

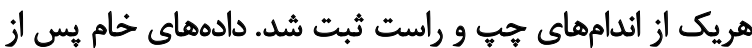

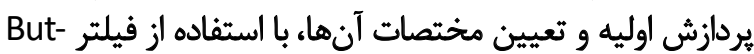

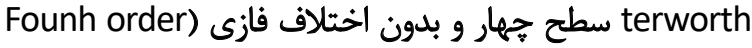
شutterworth low pass filter

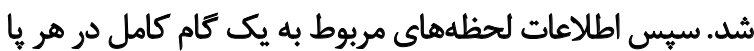

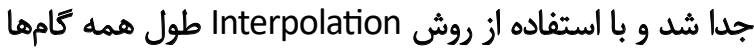

$$
\text { به ب. إدرصد تبديل شد. }
$$

يس از اجراي ييشآزمون، افراد به مدت جهار هفته و هر هفته

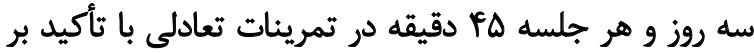

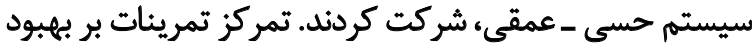

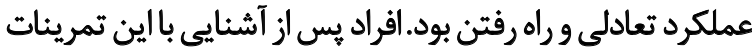

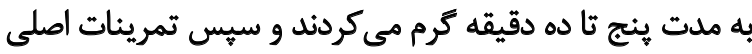

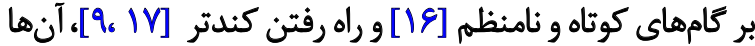

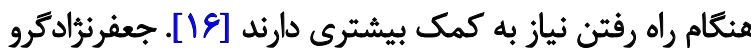

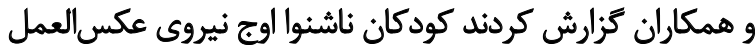

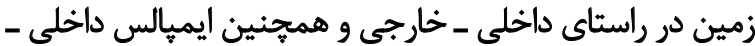

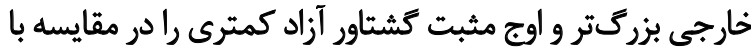

همسالان سالم طى راه رفتن دارا هستند [1/]].

مجلسى و همكار ان گزارش كردند كه فعاليت عضلات درشت فئى

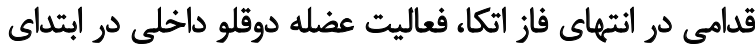

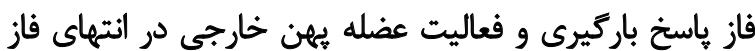

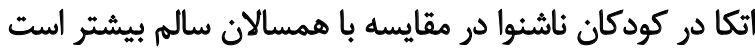

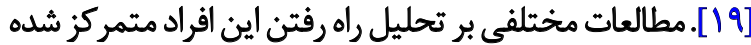

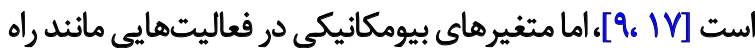

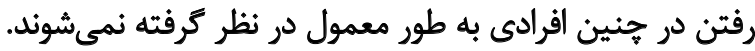

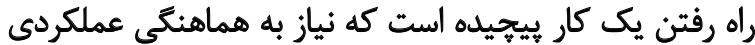

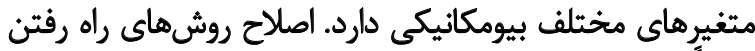

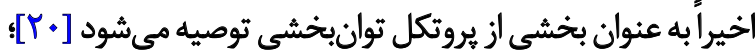

بنابراين متغيرهاى بيومكانيكى راه رفتن داراى اهميت كلينيكى برائ

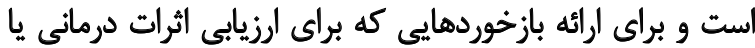

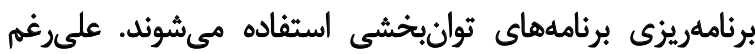

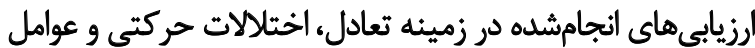

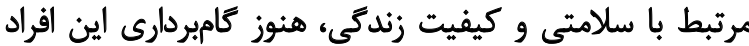

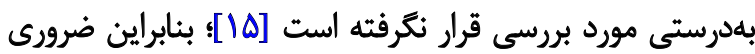

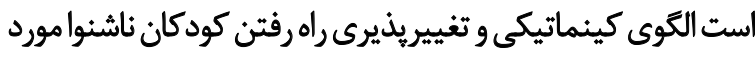

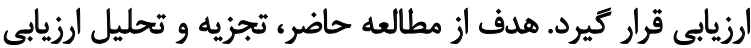

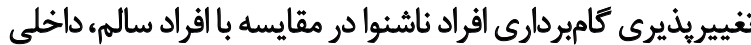

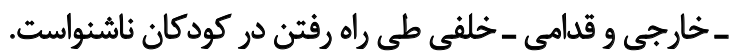

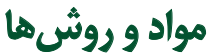

جامعه آمارى اين بثروهش افراد ناشنوا و افراد با شنوايى عادى

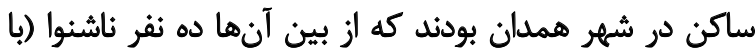

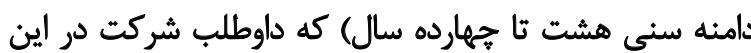

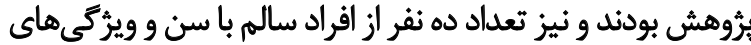

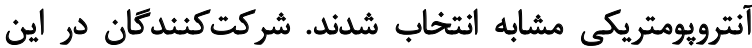

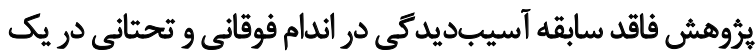

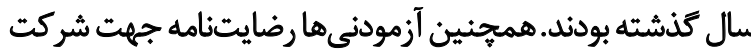

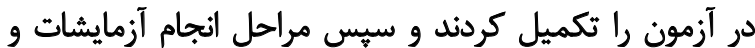

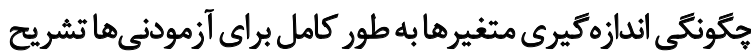

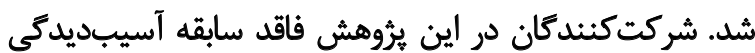

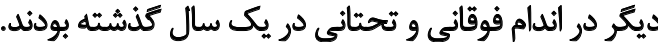

براي اندازميرى متغيرهاي كينماتيكى راه رفتن، از سيستم

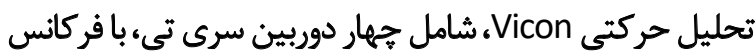

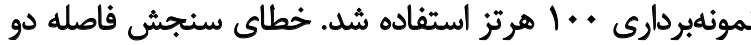

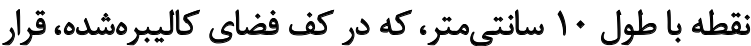




$$
\text { تغييريذيرى متغير هاى فضايى -زمانى }
$$

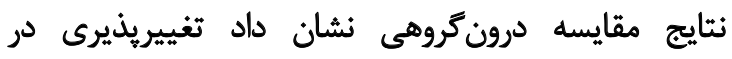

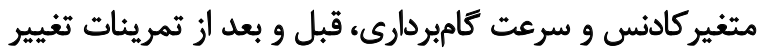

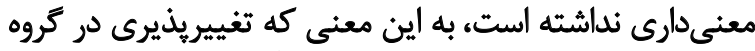

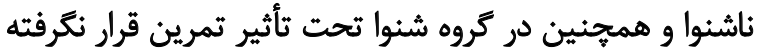

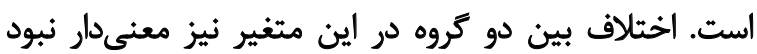

$$
\text { (جدول شماره ()). - (جناف دين }
$$

$$
\text { تغيير يذيرى مثغير هاى فضايى }
$$

تغييريذيرى در متغير طول كام و طول قدم، قبل و بعد از

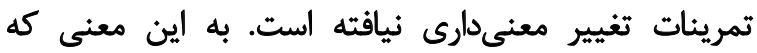

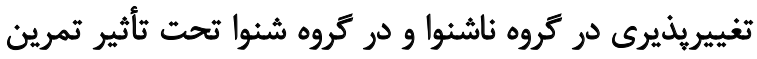

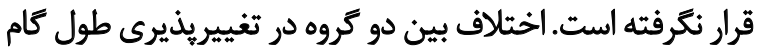

$$
\begin{aligned}
& \text { معنى دار نبود (جدول شماره T). } \\
& \text { تغيير يذيرى متغيرهاى زمائى }
\end{aligned}
$$

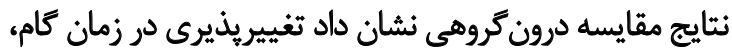

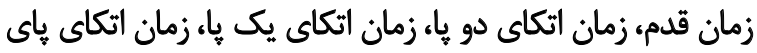

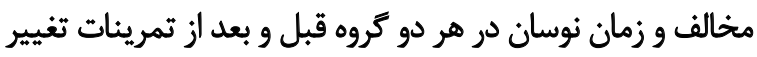

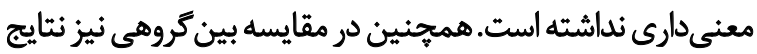

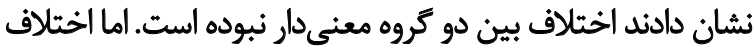

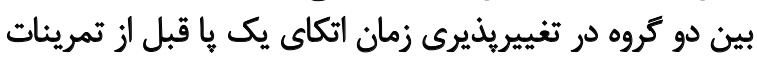

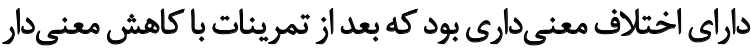

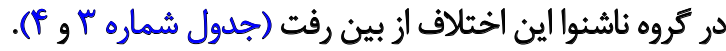

را انجام مي دادند. كيرندههاى حسى ـعمقى در كف ها واو تنه بود.

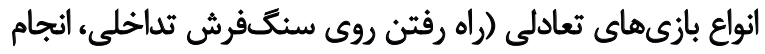

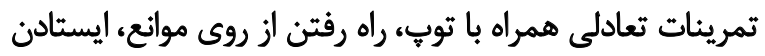

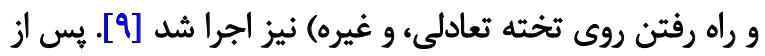
اجراى دوره تمرينى يس آزمون اجرا شدئ

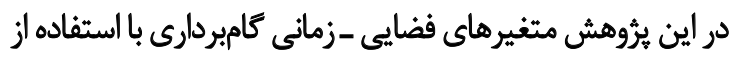

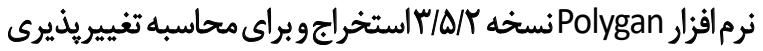
اين متغيرها از فرمول شماره ا (ضريب تغييرات) استفاده شد. نداري

$$
\operatorname{CoV}=\frac{\Sigma S D}{X} \times 100
$$

فرمول COV: ضريب تغييريذيرى كه معمولاً به شكل درصد

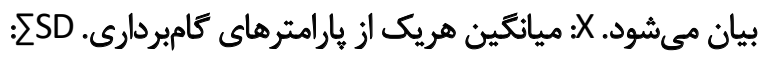

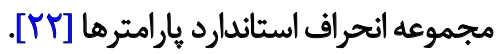
براي بررسي نرمال بودن دادهها و مكان استفاده از آزمونهاي إنهاي

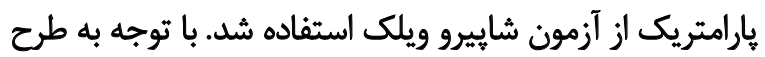

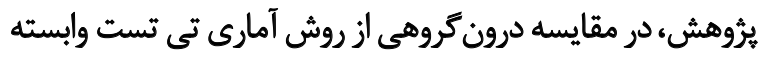

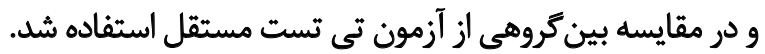

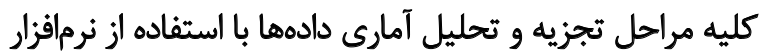

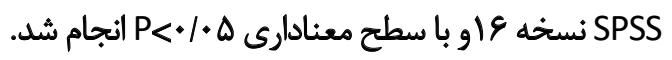

\section{نتايج}

\begin{tabular}{|c|c|c|c|c|}
\hline شاخص توده بدن (BMI) & جرم (كيلوتَّم) & قد (متر) & سن (سال) & $\operatorname{los}$ \\
\hline$|Q / \Delta|(T / F+)$ & $F \cdot 18 \cdot\left(1 T / T^{\circ}\right)$ & $I / F T(\cdot / I I)$ & $11 / 4+(1 / 94)$ & كروه ناثشنوا \\
\hline $\mid V / I+(f / \Psi \wedge)$ & $m / q \cdot(I r / \Delta \cdot)$ & $1 / 1 \% 9(+1+1)$ & $1 \cdot / f+(1 / \Delta \cdot)$ & كروه كتترل \\
\hline
\end{tabular}

ميانكين و انحراف استاندارد سن، قد، جرم و شاخص توده بدنى

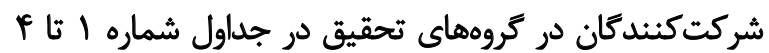

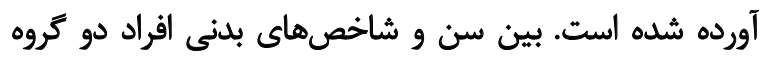

\begin{tabular}{|c|c|c|c|c|c|c|c|}
\hline Pf & Pr & PY & PI & بعد از تمرين & قبل از تمرين & كروه & متغير \\
\hline \multirow{2}{*}{.1 .8} & \multirow{2}{*}{$\cdot \pi$} & \multirow{2}{*}{.18} & \multirow{2}{*}{.$/ m$} & F/IT(I/IV) & $r / / r(1 / / q)$ & كتترل & \multirow{2}{*}{ كادنس } \\
\hline & & & & fl.r(Y/AT) & $r / T q(T / P A)$ & ناتشنوا & \\
\hline \multirow{2}{*}{ rות } & \multirow{2}{*}{.$/ 1$} & \multirow{2}{*}{.188} & \multirow{2}{*}{.$/ \pi$} & $f / \cdots(f / \cdot r)$ & $\varphi / \cdot r(\varphi / \cdot \Lambda)$ & كنترل & \multirow{2}{*}{ سرعت } \\
\hline & & & & $\checkmark / /(\checkmark / \mu)$ & $V / e f(\Delta / T \Lambda)$ & ناشنوا & \\
\hline
\end{tabular}
تفاوت معنى دارى وجود نداشت.

جدول ا. مشخصات شركت كنيدكان (عدد داخل يرائتز انحراف استاندارد است)

مجله بيومكانيك ورنش

جدول ז. نتايج مقايسه درون كروهى و بين كروهى در متغيرهاي فضايي - زمانى

مجله بيومكانيك ورنش

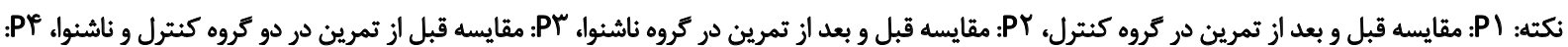

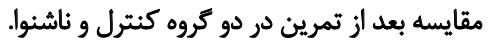


جدول r. نتايج مقايسه درون كروهى و بين كروهى در متغيرهاى فضايى ـ فضايى

\begin{tabular}{|c|c|c|c|c|c|c|c|}
\hline Pf & PY & PY & PI & بعد از تمرين & قبل از تمرين & كروه & متغير \\
\hline \multirow{2}{*}{$\cdot / \varepsilon$} & \multirow{2}{*}{.$/ 1 \Delta$} & \multirow{2}{*}{.181} & \multirow{2}{*}{.$/ M$} & $r / \Gamma r(r / N \Delta)$ & $\Gamma / \Gamma(r / V \Delta)$ & كتترل & \multirow{2}{*}{ طول كام } \\
\hline & & & & V/TE (V/q)) & $\operatorname{grr}(\Delta / \cdot V)$ & ناشنوا & \\
\hline \multirow{2}{*}{.$/}$. & \multirow{2}{*}{./Ir } & \multirow{2}{*}{.$/ \Delta 1$} & \multirow{2}{*}{.$/ M \varphi$} & $r / M(r / I V)$ & $r / V E(T / M Y)$ & كتترل & \multirow{2}{*}{ طول قدم } \\
\hline & & & & $\operatorname{sigs}(f / F))$ & $\operatorname{VIgr}(\varepsilon / q 4)$ & نالشنوا & \\
\hline
\end{tabular}

مجله بيومكانيك ورزشـ

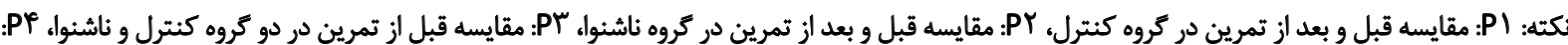

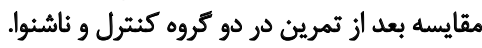

جدول F. تئايج مقايسه درون كروهى و بين كروهى در متغيرهاى زمانى

\begin{tabular}{|c|c|c|c|c|c|c|c|}
\hline Pf & Pr & PY & PI & بعد از تمرين & قبل از تمرين & كروه & متغير \\
\hline \multirow{2}{*}{.1 .9} & \multirow{2}{*}{$+/ 18$} & \multirow{2}{*}{$t / v$} & \multirow{2}{*}{.$/ M f$} & $r / 1 q(1 / T Y)$ & $r / M(M / r))$ & كتترل & \multirow{2}{*}{ زمان كام } \\
\hline & & & & $r / Q \Lambda(T / \Delta F)$ & $r / F \&(r / \Delta)$ & ناشنوا & \\
\hline \multirow{2}{*}{.$/ \cdot v$} & \multirow{2}{*}{.1 .8} & \multirow{2}{*}{$\cdot|N|$} & \multirow{2}{*}{.$/ M$} & $r / \Lambda \Delta(F / Y F)$ & $r / V \Delta(T / ৭ q)$ & كتترل & \multirow{2}{*}{ زمان قدم } \\
\hline & & & & $\varepsilon / 9(T / M)$ & $\operatorname{SISV}(T / \Delta S)$ & ناشنوا & \\
\hline \multirow{2}{*}{./lf } & \multirow{2}{*}{$\%$} & \multirow{2}{*}{.$/ 19$} & \multirow{2}{*}{.$/ 4$} & $r / 9 f(r / M q)$ & $m / I V(m / M e)$ & كتترل & \multirow{2}{*}{ زمان اتيكاي يك } \\
\hline & & & & $\operatorname{siNV}\left(T / M^{\prime}\right)$ & S/MA (W/gT) & ئاشنوا & \\
\hline \multirow{2}{*}{. } & \multirow{2}{*}{.$/ p q$} & \multirow{2}{*}{.$/ K A$} & \multirow{2}{*}{.$/ \mu \varphi$} & $18 / 9(11 / 98)$ & $19 / 1 P 1(11 / 94)$ & كتترل & \multirow{2}{*}{ زمان اتكايى دو } \\
\hline & & & & re/ar (Ir/Re) & IVEV (NQE) & ثاشنوا & \\
\hline \multirow{2}{*}{. $/ \Delta r$} & \multirow{2}{*}{.$|\Delta|$} & \multirow{2}{*}{. /NA } & \multirow{2}{*}{.$/ \mu$} & $r / Q P(T / Q Y)$ & $r / g q(r / \cdot r)$ & كتنرل & \multirow{2}{*}{ زمان اتكاي ياى } \\
\hline & & & & $P / T r(I / T F)$ & PRA (T/MP) & ناشنوا & \\
\hline \multirow{2}{*}{$\cdot / \Delta \Delta$} & \multirow{2}{*}{$\cdot / \mu$} & \multirow{2}{*}{.$/ 109$} & \multirow{2}{*}{.$/ N F$} & YNE (TY/NT) & TNVA(TY/N) & كتترل & \multirow{2}{*}{ زمان نوسان } \\
\hline & & & & $T / \Delta(1 S / \Delta Q)$ & Wa (NA) & ناشنوا & \\
\hline
\end{tabular}

مجله بيومكانيكورزش

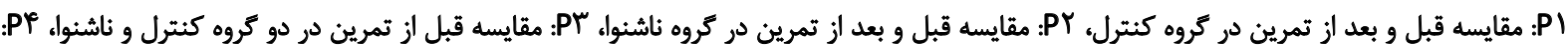
مقايسه بعد از تمرين در دو كروه كنترل و ناشنوا.

تعادل كه ممكن است مانع اجراي ايدهآل در اين كودكان شودا،

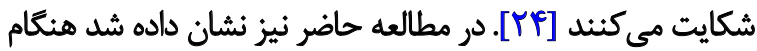

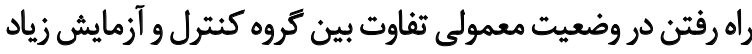

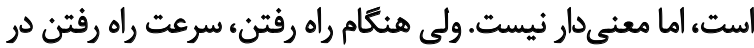

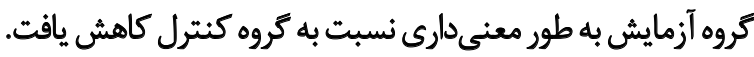
مطابق با نتايج، تمرينات تعادلى حسى ـ عمقى نتوانسته بود

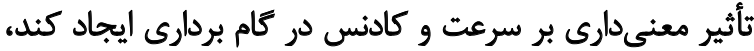

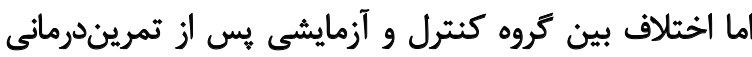

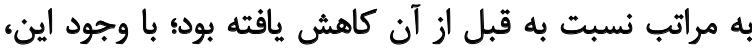

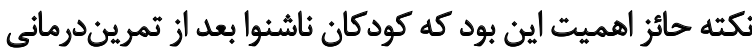

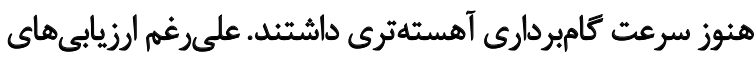

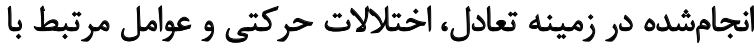
سلامتى و كيفيت زندگى، هنوز برنامه آموزشى براى اين كودكاني

هدف اين مطالعه ارزيابي تغييريذيرى كامبرداري افراد ناشنوا و

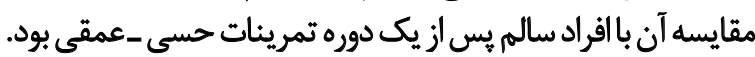

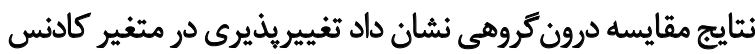

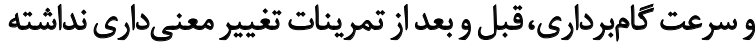

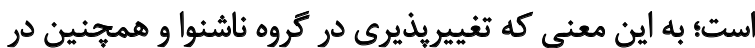
كروه شنوا تحث تأثير تمرين قرار نتخرفته است

اختلاف بين دو كروه در اين متغير نيز معنى دار نبود. در اغلب

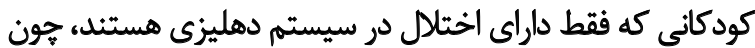

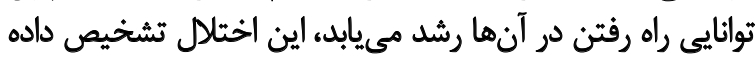

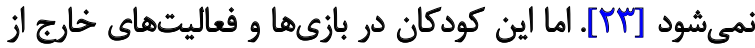

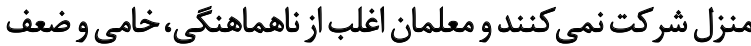


قامت و متوقف شدن تأخير حركتى ايجاد شده بود [11]. تغييرات

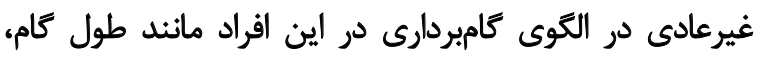

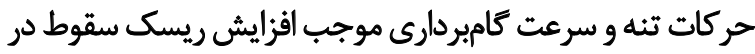

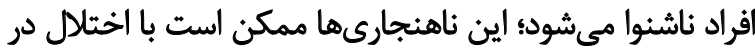

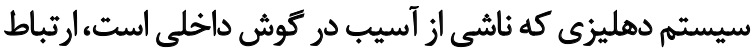

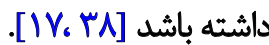

\section{تتيجلئيرىنهايي}

نتايج مطالعه حاضر نشان دادند تغييريذيرى بر اثر تمرينات

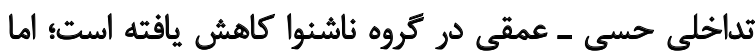

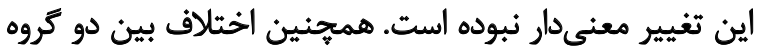

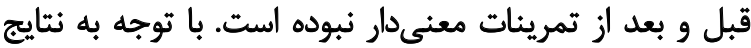

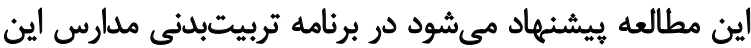

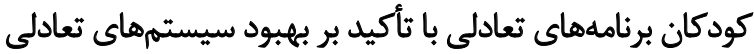
بلهويره سيستم حسى -عمقى كنجانده شودي

ملاحظات اخلاقي - اتي

$$
\text { ييروى از اصول اخلاق يثروهش }
$$

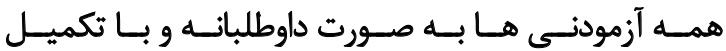

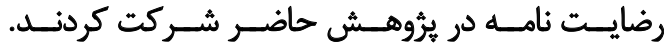

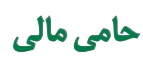

اين مقاله حامى مالى نداشته است.

$$
\text { مشاركت ثويسند }
$$

همه نويسندكان در آماده سازى مقاله مشاركت داشته اند.

$$
\text { تعارض مناقع }
$$

بنابر اظهار نويسندكان اين مقاله تعارض منافع ندارد.

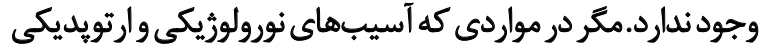

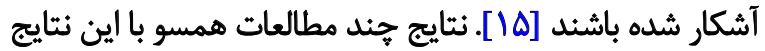

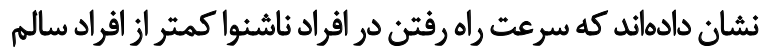

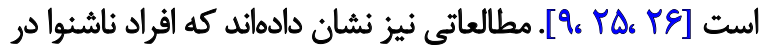

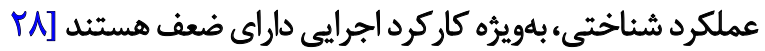

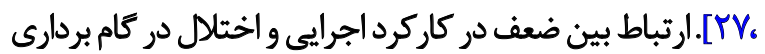

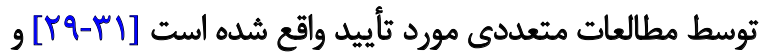
الين موضوع در راه رفتن با تكليف دوكانه واضحتر است.

مطابق با نتايج برؤهش حاضر، تغييريذيرى در متغير طول

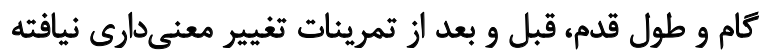

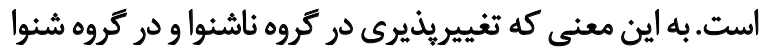

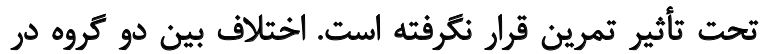

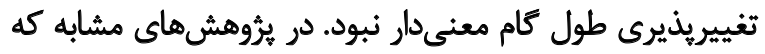

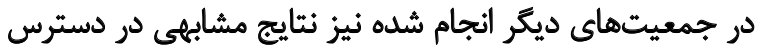

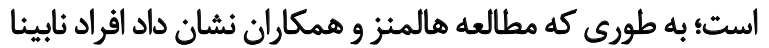

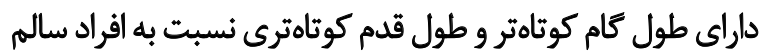

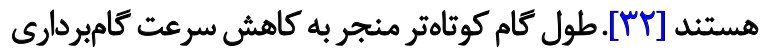

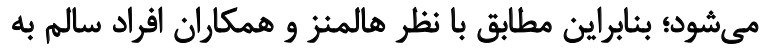

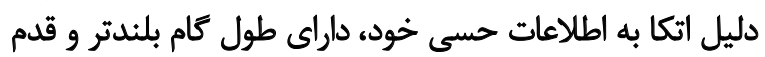

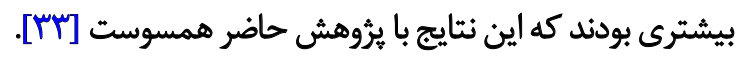

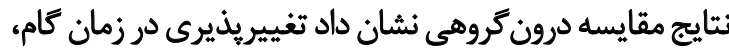

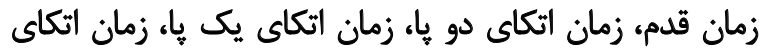

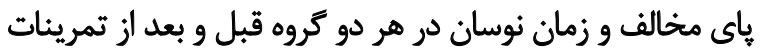

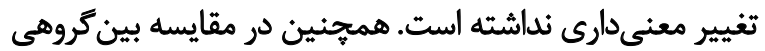

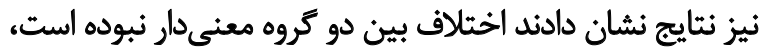

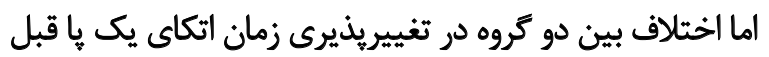

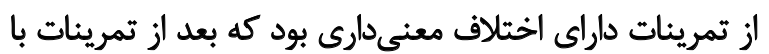

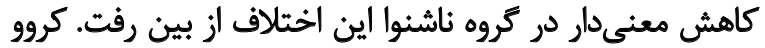

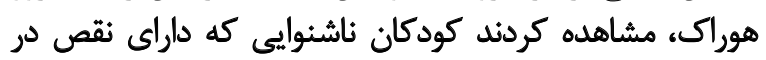

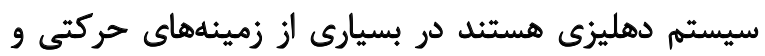

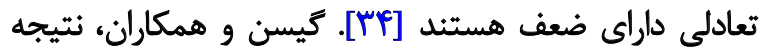
كاشت حلزون را بر توانايىهاى حركتى كوديتي

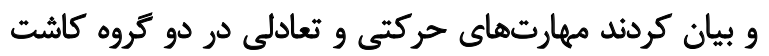

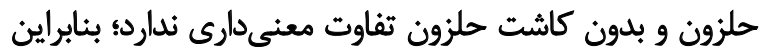

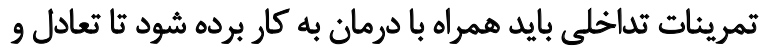

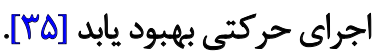

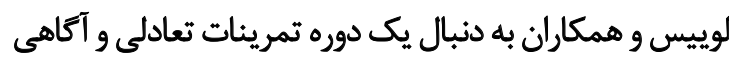

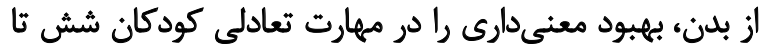

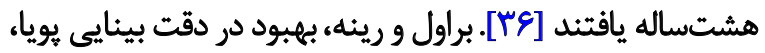

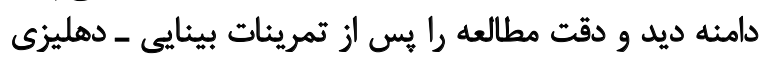

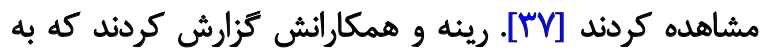

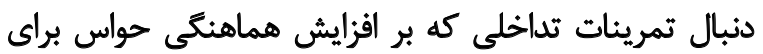

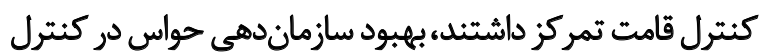




\section{References}

[1] Cruickshanks KJ, Wiley TL, Tweed TS, Klein BE, Klein R, Mares-Perlman $\mathrm{JA}$, et al. Prevalence of hearing loss in older adults in Beaver Dam, Wisconsin: The epidemiology of hearing loss study. Am J Epidemiol. 1998; 148(9):879-86. [DOI:10.1093/oxfordjournals.aje.a009713] [PMID]

[2] Davis AC. The prevalence of hearing impairment and reported hearing disability among adults in Great Britain. Int J Epidemiol. 1989; 18(4):9117. [DOI:10.1093/ije/18.4.911] [PMID]

[3] World Health Organization. Deafness and hearing loss. Geneva: World Health Organization; 2020. https://www.who.int/news-room/factsheets/detail/deafness-and-hearing-loss

[4] Carvill S. Sensory impairments, intellectual disability and psychiatry. J Intellect Disabil Res. 2001; 45(Pt 6):467-83. [DOI:10.1046/j.13652788.2001.00366.x] [PMID]

[5] Mohr PE, Feldman JJ, Dunbar JL, McConkey-Robbins A, Niparko JK, Rittenhouse RK, et al. The societal costs of severe to profound hearing loss in the United States. Int J Technol Assess Health Care. 2000; 16(4):112035. [DOI:10.1017/S0266462300103162] [PMID]

[6] Wiener-Vacher SR. Vestibular disorders in children. Int J Audiol. 2008; 47(9):578-83. [DOI:10.1080/14992020802334358] [PMID]

[7] Koffler T, Ushakov K, Avraham KB. Genetics of hearing loss: Syndromic. Otolaryngol Clin North Am. 2015; 48(6):1041-61. [DOI:10.1016/j. otc.2015.07.007] [PMID] [PMCID]

[8] De Kegel A, Dhooge I, Cambier D, Baetens T, Palmans T, Van Waelvelde $\mathrm{H}$. Test-retest reliability of the assessment of postural stability in typically developing children and in hearing impaired children. Gait posture. 2011; 33(4):679-85. [DOI:10.1016/j.gaitpost.2011.02.024] [PMID]

[9] Majlesi M, Farahpour N, Azadian E, Amini M. The effect of interventional proprioceptive training on static balance and gait in deaf children. Res Dev Disabil. 2014; 35(12): 3562-7. [DOI:10.1016/j.ridd.2014.09.001] [PMID]

[10] Potter CN, Silverman LN. Characteristics of vestibular function and static balance skills in deaf children. Phys Ther. 1984; 64(7):1071-5. [DOI:10.1093/ptj/64.7.1071] [PMID]

[11] Rine RM, Braswell J, Fisher D, Joyce K, Kalar K, Shaffer M. Improvement of motor development and postural control following intervention in children with sensorineural hearing loss and vestibular impairment. Int J Pediatr Otorhinolaryngol. 2004; 68(9):1141-8. [DOI:10.1016/j. ijporl.2004.04.007] [PMID]

[12] Jafari Z, Malayeri SA. The effect of saccular function on static balance ability of profound hearing-impaired children. Int J Pediatr Otorhinolaryngol. 2011; 75(7):919-24. [DOI:10.1016/j.ijporl.2011.04.006] [PMID]

[13] Melo RdS, Silva PWAd, Silva LVCd, Silva Toscano CFd. Postural evaluation of vertebral column in children and teenagers with hearing loss. Intl Arch Otorhinolaryngol. 2011; 15(2):195-202. [DOI:10.1590/S180948722011000200012]

[14] Angeli S. Value of vestibular testing in young children with sensorineural hearing loss. Arch Otolaryngol Head Neck Surg. 2003; 129(4):478-82. [DOI:10.1001/archotol.129.4.478] [PMID]

[15] Rajendran V, Roy FG, Jeevanantham D. Postural control, motor skills, and health-related quality of life in children with hearing impairment: A systematic review. Eur Arch Otorhinolaryngol. 2012; 269(4):1063-71. [DOI:10.1007/s00405-011-1815-4] [PMID]
[16] Melo RdS, Silva PWAd, Tassitano RM, Macky CFS, Silva LVCd. Balance and gait evaluation: Comparative study between deaf and hearing students. Rev Paul Pediatr. 2012; 30(3):385-91. [DOI:10.1590/S010305822012000300012]

[17] Li L, Simonsick EM, Ferrucci L, Lin FR. Hearing loss and gait speed among older adults in the United States. Gait Posture. 2013; 38(1):25-9. [DOI:10.1016/j.gaitpost.2012.10.006] [PMID] [PMCID]

[18] Jafarnezhadgero AA, Majlesi M, Azadian E. Gait ground reaction force characteristics in deaf and hearing children. Gait posture. 2017; 53:23640. [DOI:10.1016/j.gaitpost.2017.02.006] [PMID]

[19] Majlesi M, Azadian E, Farahpour N, Jafarnezhad AA, Rashedi H. Lowe limb muscle activity during gait in individuals with hearing loss. Australas Phys Eng Sci Med. 2017; 40(3):659-65. [DOI:10.1007/s13246-0170574-y] [PMID]

[20] Heiderscheit B. Gait retraining for runners: In search of the ideal. J Orthop Sports Phys Ther. 2011; 41(12):909-10. [DOI: 10.2519/ jospt.2011.0111] [PMID]

[21] Winter DA. Biomechanics and motor control of human movement. Hoboken: John Wiley \& Sons; 2009. [DOI:10.1002/9780470549148]

[22] Azadian E, Taheri HR, Saberi Kakhki AR, Farahpour N. [Effects of dual-tasks on spatial-temporal parameters of gait in older adults with impaired balance (Persian)]. Iran J Ageing. 2016; 11(1):100-9. [DOI:10.21859/sija-1101100]

[23] Rine RM. Growing evidence for balance and vestibular problems in children. Audiol Med. 2009; 7(3):138-42. [DOI:10.1080/16513860903181447]

[24] Butterfield SA. Gross motor profiles of deaf children. Percept Mot Skills. 1986; 62(1):68-70. [DOI:10.2466/pms.1986.62.1.68] [PMID]

[25] Cavanaugh JT, Goldvasser D, McGibbon CA, Krebs DE. Comparison of head-and body-velocity trajectories during locomotion among healthy and vestibulopathic subjects. J Rehabil Res Dev. 2005; 42(2):191-8. [DOI:10.1682/JRRD.2004.01.0005] [PMID]

[26] Marchetti GF, Whitney SL, Blatt PJ, Morris LO, Vance JM. Tempora and spatial characteristics of gait during performance of the Dynamic Gait Index in people with and people without balance or vestibular disorders. Phys Ther. 2008; 88(5):640-51. [DOI:10.2522/ptj.20070130] [PMID] [PMCID]

[27] Lin FR, Ferrucci L, Metter EJ, An Y, Zonderman AB, Resnick SM. Hearing loss and cognition in the Baltimore Longitudinal Study of Aging. Neuropsychology. 2011; 25(6):763-70. [DOI:10.1037/a0024238] [PMID] [PMCID]

[28] Tun PA, McCoy S, Wingfield A. Aging, hearing acuity, and the attentional costs of effortful listening. Psychol Aging. 2009; 24(3):761-6. [DOI:10.1037/a0014802] [PMID] [PMCID]

[29] Allali G, Assal F, Kressig RW, Dubost V, Herrmann FR, Beauchet O. Impact of impaired executive function on gait stability. Dement Geriatr Cogn Disord. 2008; 26(4):364-9. [DOI:10.1159/000162358] [PMID]

[30] Ble A, Volpato S, Zuliani G, Guralnik JM, Bandinelli S, Lauretani F, et al. Executive function correlates with walking speed in older persons: The InCHIANTI study. J Am Geriatr Soc. 2005; 53(3):410-5. [DOI:10.1111/ j.1532-5415.2005.53157.x] [PMID]

[31] Sheridan PL, Solomont J, Kowall N, Hausdorff JM. Influence of executive function on locomotor function: Divided attention increases gait variability in Alzheimer's disease. J Am Geriatr Soc. 2003; 51(11):1633-7. [DOI:10.1046/j.1532-5415.2003.51516.x] [PMID] 
[32] Hallemans A, Ortibus E, Truijen S, Meire F. Development of independent locomotion in children with a severe visual impairment. Res Dev Disabil. 2011; 32(6):2069-74. [DOI:10.1016/j.ridd.2011.08.017] [PMID]

[33] Hallemans A, Ortibus E, Meire F, Aerts P. Low vision affects dynamic stability of gait. Gait Posture. 2010; 32(4):547-51. [DOI:10.1016/j.gaitpost.2010.07.018] [PMID]

[34] Crowe TK, Horak FB. Motor proficiency associated with vestibular deficits in children with hearing impairments. Phys Ther. 1988; 68(10):14939. [PMID]

[35] Gheysen F, Loots G, Van Waelvelde H. Motor development of deaf children with and without cochlear implants. J Deaf Stud Deaf Educ. 2008; 13(2):215-24. [DOI:10.1093/deafed/enm053] [PMID]

[36] Lewis S, Higham L, Cherry DB. Development of an exercise program to improve the static and dynamic balance of profoundly hearingimpaired children. Am Ann Deaf. 1985; 130(4):278-84. [DOI:10.1353/ aad.2012.1020] [PMID]

[37] Braswell J, Rine RM. Preliminary evidence of improved gaze stability following exercise in two children with vestibular hypofunction. Int J Pediatr Otorhinolaryngol. 2006; 70(11):1967-73. [DOI:10.1016/j. ijporl.2006.06.010] [PMID]

[38] Melo RdS, Silva PWAd, Tassitano RM, Macky CFST, Silva LVCd. Avaliação do equilíbrio corporal e da marcha: Estudo comparativo entre surdos e ouvintes em idade escolar. Rev Paul Pediatr. 2012; 30(3):38591. [DOI:10.1590/S0103-05822012000300012] 